\title{
Gestión del conocimiento en torno a víctimas de minas antipersonal (MAP), en el marco del conflicto armado en Colombia: Análisis tendencial desde la bibliometría y Tree of Science
}

\author{
Clara Isabel López Gualdrón ${ }^{\mathrm{a}}$, Catalina Ortega Zambrano ${ }^{\mathrm{b}}$, Ruth Zárate Rueda ${ }^{\mathrm{c}}$ \\ ${ }^{a}$ Doctora (C) Ingeniería, Aréa Gestión Tecnológica, Universidad Industrial de Santander, \\ Bucaramanga, Colombia. Clalogu@uis.edu.co ${ }^{\mathrm{b}}$ Magíster en Derechos Humanos, Universidad \\ Industrial de Santander, Bucaramanga, Colombia, cattazambrano11@outlook.es. ${ }^{\mathrm{c}}$ Doctora en \\ Educación, Universidad Industrial de Santander, Bucaramanga, Colombia, \\ ruthzaraterueda@,uis.edu.co
}

\section{Resumen}

Al hablar del conflicto armado colombiano, se evoca a las personas históricamente afectadas por los hechos victimizantes acaecidos durante más de cinco décadas en el país. Estos sucesos han menoscabado los Derechos Humanos (DDHH) de las víctimas durante este periodo. Una acción que se utiliza generalmente como método de guerra, por actores armados estatales y no estales, son las Minas Antipersonal (MAP) la cual genera discapacidad permanente en los sobrevivientes. Por consiguiente, se requieren procesos de rehabilitación integral desde diferentes disciplinas que contribuyan a las personas a adaptarse a su nueva condición. El propósito de este artículo consiste en identificar la producción de conocimiento cientifico relacionada con las estrategias de Rehabilitación Basada en Comunidad (RBC), rehabilitación en procesos de amputación o lesiones asociadas a víctimas de MAP y métodos innovadores de rehabilitación presentes en las áreas de la Salud, Psicología, Artes, Ciencias Sociales y Humanas en bases de datos de alto impacto. La metodología se fundamentó en el uso de software el cual fue Vantage Point para la organización y análisis de volúmenes de información; asimismo, se implementó la herramienta web Tree of Science (ToS) para la identificación de documentos seminales no registrados en la fase de revisión de datos previa. Finalmente, los resultados señalan las tendencias en investigación en producción en la temática de estudio, enfatizando en año, país, autor, campo de acción, filiación institucional, colaboración entre autores, palabras claves, palabras claves investigadoras, citación por artículo en la temática objeto de estudio. Los resultados demuestran el 
proceso de gestión de conocimiento en torno a víctimas de MAP, en el marco del conflicto armado.

Palabras clave: Gestión del conocimiento, víctimas de Minas Antipersonal, Tree of Science, conflicto armado, bibliométria.

\section{Introducción}

El trabajo científico y la producción de resultados de investigación se encuentra condicionado a un medio caracterizado por la medición de la productividad y la necesidad de establecer el grado de pertinencia de aquello que se investiga por las diversas comunidades científicas, así como lo que se difunde. En este contexto emerge el análisis bibliométrico que, está constituido por la aplicación de métodos, herramientas e indicadores con el objeto de analizar grandes conjuntos de datos e información relacionada con la producción científica y tecnológica (Ordoñez et al, 2009), además de instrumentos capaces de evidenciar hasta dónde las comunidades científico - académicas o las personas influyen con sus desarrollos a sus semejantes en el medio en el que realizan sus actividades de Ciencia, Tecnología e Innovación (CTI).

Los datos cuantitativos suministrados por la literatura científica y técnica, basados en el análisis estadístico, sirven para evaluar la actividad científica mediante indicadores bibliométricos. De tal forma, se emplean por un lado indicadores para analizar el tamaño, crecimiento y distribución de la bibliografía científica (libros, revistas, patentes), cuyo propósito es mejorar las actividades de información, documentación y comunicación científica, de otra parte, para analizar los procesos de generación, divulgación y uso de la literatura científica.

Con base en la tesis de Pritchard (1969), se entiende por bibliometría a la ciencia que estudia la naturaleza y trayectoria de una disciplina por medio de la sistematización y análisis de las distintas facetas de la comunicación escrita y, por otro lado, también indica que la metodología bibliométrica se ocupa del análisis cuantitativo de determinadas variables presentes en un texto publicado, con el fin de aproximarse a la situación de un campo científico en un tiempo determinado.

\section{Metodología}

El presente artículo desde la metodológica comprendió en primera instancia la fase de análisis bibliométrico, en donde no se encontró producción de conocimiento científico 
relacionada con las estrategias de Rehabilitación Basada en Comunidad (RBC), rehabilitación en procesos de amputación o lesiones asociadas a víctimas de MAP y métodos innovadores de rehabilitación desde las áreas de la Salud, Psicología, Artes, Ciencias Sociales y Humanas, por tanto, surgió la necesidad de realizar un análisis apoyado en la herramienta web Tree of Science $(\mathrm{ToS})^{1}$ el cual permitió ahondar en los artículos seminales, estructurales y las publicaciones contemporáneas en la temática estudiada (Figura 1).

Con el propósito de profundizar en la búsqueda de artículos que no se encontraron en la fase metodológica del análisis Bibliométrico, se utilizó la herramienta web Tree of Science, la cual fue fundamental en la selección de artículos relevantes en la identificación de procesos de rehabiitación integral con víctimas de MAP. Estos se plasman en forma de árbol (Figura 1), en donde los artículos clásicos o seminales se representan en la raíz (color amarillo), los artículos que le dan estructura al conocimiento son los que se encuentran en el tronco (color café) y las hojas son los artículos más recientes (color verde).

En total se analizaron 46 artículos los cuales en primera instancia se buscaron en la base de datos Web of Science, a partir de una ecuación de búsqueda (Tabla 1), la cual se construyó como resultado de la combinación de las diferentes expresiones de búsqueda (Tabla 1) y operadores boléanos "AND" y "OR", en el cual "AND” cumplió la función de localizar artículos que tuvieran todos los términos de búsqueda especificados y "OR" de localizar registros que conteuvieran un término o sinónimos del mismo (Dodge, 2009).

Una vez culminada la fase de filtración de documentos, se procedió a elaborar una matriz de clasificación en el programa Excel, versión 2016 para Windows 7, en la cual se especificaron las siguientes categorías: título, autor, año de publicación, filiación institucional, palabras clave y abstracts. Adicionalmente se incluyeron dos categorías de interés para el investigador: campos y palabras clave investigadoras; dichas categorías se consideraron pertinentes, por cuanto -al efectuar la revisión de los abstract- se encontró que los artículos planteaban diferentes temáticas en relación a procesos de rehabilitación integral con víctimas de MAP; así se contrastó la producción literaria respecto al tema de estudio y la orientación de estas prácticas a la salud, el componente psicosocial, educación, y lo técnico.

Posteriormente, la matriz de clasificación se ingresó en el software Vantage Point. Este Software permitió la organización de la información mediante el uso de métodos cuantitativos y estadísticos, representados gráficamente en tablas, adunas y nubes de

\footnotetext{
${ }^{1}$ La filosofía de ToS se ha fundamentado en tres pilares: simplicidad, efectividad e innovación. La simplicidad se encuentra en los conceptos orgánicos para facilitar al usuario entender la estructura de la ciencia a partir de la metáfora del árbol. La efectividad se basa en la precisión de los resultados obtenidos de los artículos científicos. Por último, la innovación parte de un mejoramiento continuo de los servicios prestados de tal manera que pueda sorprender a los usuarios (SIRY, 2018).
} 
palabras que evidenciaron las tendencias bibliográficas existentes en el grupo de publicaciones científicas seleccionado.

Para hacer uso de la herramienta ToS, se procedió a descargar los registros mediante EndNote, en donde se escribieron los registros que se deseaban seleccionar. Finalmente, se cambió el formato de archivo con el objetivo de obtener un archivo en texto plano e ingresarlos a la herramienta web para la selección inteligente de artículos cientificos aplicando el análisis de redes. Los resultados de los estudios más significativos se presentan a continuación.

\section{Resultados}

Dentro de los artículos seminales se encontró el estudio titulado $A$ New Therapeutic Community: Development of a Compassion-Focussed and Contextual Behavioural Environment, en donde Veale et al. (2014), pone de manifiesto el apoyo significiativo que tiene para una persona el terner una red de apoyo terciaria que lo acoja, por cuanto las relaciones sociales y las comunidades proporcionan el contexto y el ímpetu para una variedad de desarrollos psicológicos, desde la expresión genética hasta el desarrollo de autoidentidades centrales (Veale et al., 2014). Lo anterior genera la necesidad de pensar en los cambios y procesos terapéuticos que ocurren dentro de un contexto comunitario y cómo las comunidades pueden permitir el cambio terapéutico, es decir, este planteamiento expone la relevancia de incluir la red terciaria en los procesos de rehabilitación de las víctimas de MAP, tomando al indivduo como un todo y no de manera fragmentada. La red de apoyo social tiene un rol protagónico en los contextos de rehabiliación, toda vez que son estas las que potencian desde lo psicosocial y emocional el proceso de la víctima de MAP.

Es de resaltar la mención especial que se realiza en el documento sobre la investigación de la naturaleza de los sistemas de regulación de emociones evolucionadas, la forma en que las relaciones sociales conllevan dichos sistemas, la importancia de las emociones afiliativas en la regulación de la amenaza, lo anterior, fortalece la necesidad de incorporar dentro de las estrategias de rehabilitación en procesos de amputación o lesiones asociadas a víctimas de MAP $\mathrm{y}$ métodos innovadores de rehabilitación el componente psicosocial como elemento vital para generar procesos de rehabilitación holísticos innovadores, que como lo menciona Veale et al. (2014), debe integrar la red terciaria como elemento de acompañamiento y educación en el desarrollo del proceso. 
- En los artículos estructurales, se resalta el estudio realizado por Walsh \& Walsh (2003), Blast injury; Cost of illness; Economic aspect; Health care cost; Landmine; Length of stay; Limb prosthesis; Major clinical study; Social aspect; Victim; weapon, aquí se realiza un análisis histográfico sobre las MAP, las afectaciones permanentes y su uso en el marco de contextos de conflicto contra actores civiles y armados. El autor resalta que, las víctimas de MAP utilizan una cantidad significativa de recursos médicos; resaltando un hecho sistemático, la precariedad económica de las regiones donde ocurren los incidentes que, son países sin una infraestructura médica sofisticada y con recursos limitados, donde la rehabilitación es difícil en las mejores circunstancias. Se sugiere que solo una cuarta parte de los pacientes con amputación secundaria a minas terrestres reciben la atención adecuada.

Lo anterior refuerza la necesidad de plantear un proceso de rehabilitación integral que se adapte a los contextos en donde ocurren los accidentes e incidentes con MAP, es decir, procesos idóneos para que los segmentos poblacionales afectados no tengan que desplazarse a las urbes a recibir atención médica, si bien el autor resalta las carencias socioeconómicas de los entornos, no plantean alternativas de solución para mitigar las consecuencias de no realizar procesos de rehabilitación adecuados con la población víctima de MAP en los tiempos que correponde desde todos los componentes, técnico, físico y psicosocial.

Dentro de los artículos contemporanéos se resaltar el trabajo realizado por Isaacson et al. (2017), The center for rehabilitation sciences research: Advancing the rehabilitative care for service members with complex trauma, el Centro de Investigación de Ciencias de la Rehabilitación (CRSR) sistematizó las prácticas que han venido desarrollando para avanzar en la atención de rehabilitación para los miembros del servicio con lesiones relacionadas con el combate, entre estas MAP, pacientes con complicaciones ortopédicas, cognitivas y neurológicas. Se describe el apoyo a proyectos de investigación exhaustivos para optimizar las estrategias de tratamiento y promover el regreso exitoso al deber y la reintegración comunitaria de los miembros del servicio lesionados.

Esta estrategia es fundamental para tomar algunos lineamientos en procesos de rehabilitación con civiles, por cuanto plantea cuatro áreas de investigación: (1) identificar barreras para la rehabilitación y reintegración exitosas, (2) mejorar las estrategias de manejo del dolor para promover la plena participación en programas de rehabilitación, (3) aplicar nuevas tecnologías para avanzar en métodos de rehabilitación y mejorar evaluaciones de resultados, y (4) transferir nuevas tecnologías para mejorar la capacidad funcional, la independencia y la calidad de vida. Cada una de estas áreas de investigación trabaja articuladamente para mejorar la calidad de vida de las personas. 


\section{Conclusiones}

El análisis descrito deja de manifiesto que en la exahustiva revisión realizada desde la bibliometría, la herramienta web Tree of Science y minería de texto no se encontraron estudios que tuvieran como eje metodológico la Rehabilitación Basada en Comunidad, la cual desde sus cinco componentes (salud social, educación, empoderamiento y subsistencia), plantea alternativas de rehabilitación extrapolandóla al contexto de las víctimas de MAP. De otra parte, exceptuando la sistematización de experiencia realizada por Isaacson et al. (2017), la cual no tiene en cuenta en el articulado proceso de rehabilitación que realizan el componente psicosocial, no se encontró otro estudio que incorporar diversas áreas de conocimiento con víctimas civiles.

Se encontró que solo el 7.1\% (54 artículos), de los 758 artículos arrojados inicialmente por la ecuación de búsqueda en la base de datos Scopus sobre la temática de estudio, son relevantes y pertinentes para la presente investigación. La mayor producción científica acerca de estrategias de RBC, rehabilitación en amputación o lesiones asociadas a víctimas de MAP y métodos innovadores de rehabilitación por regiones del mundo se concentra principalmente en América del Norte con Estados Unidos y Canadá, seguido de Medio Oriente con Israel, Irán, Pakistán, Catar y Afganistán; Europa con países bajos, Dinamarca, Eslovenia y Suecia, y por último Asia con Sir Lanka y Corea del Sur, Cabe señalar que no se registra producción científica en América del Sur respecto al tema de estudio.

Se evidencia que los artículos de rehabilitación de víctimas de MAP o MUSE, registran una mayor tendencia hacia las prácticas médicas, métodos y tratamientos, así como de la combinación de estos con prácticas comunitarias que contribuyen al fortalecimiento del individuo y de su grupo familiar, para la superación de traumas de guerra y reintegración social. El campo o área en el cual se registra mayor producción científica es Salud, por lo que se puede concluir que en su mayoría los artículos se abordan la rehabilitación desde una perspectiva médica generando aportes relevantes respecto al tratamiento y rehabilitación física de víctimas de MAP o MUSE, seguido del área de fortalecimiento orientado a la superación de traumas, soporte social y emocional, no obstante también se observa que la menor contribución o aportes científicos se presentan en las áreas de subsistencia y educación. 


\section{Figuras}

Las figuras que se presentan a continuación, se han mencionado en el desarrollo del documento.

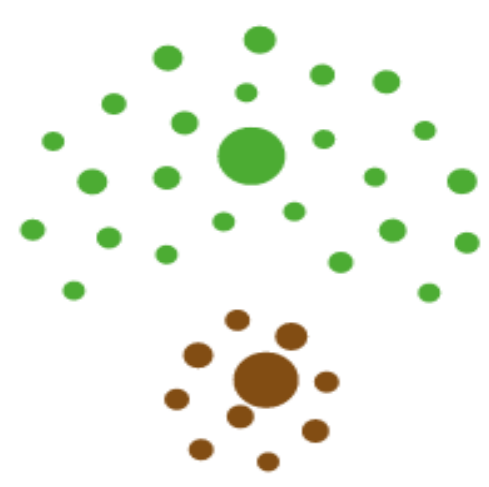

Figura 1. Árbol de la ciencia en torno a procesos de rehabilitación integral con víctimas de MAP.

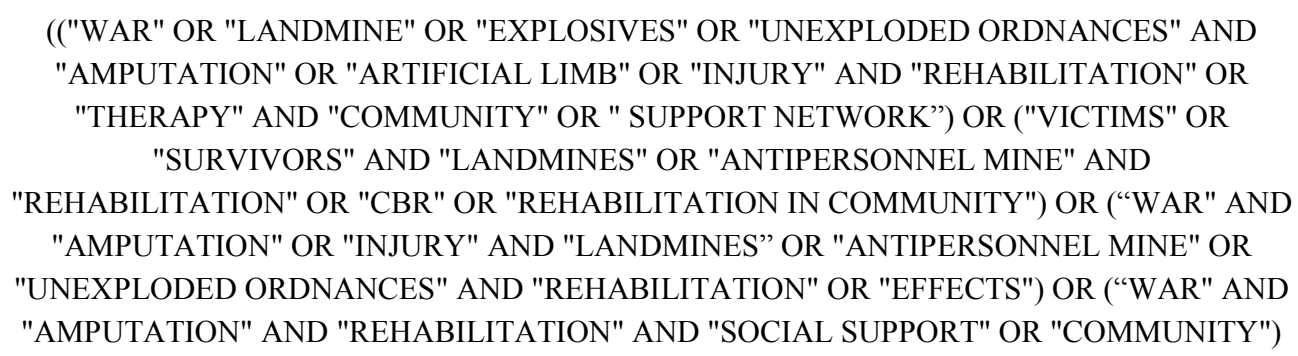

(("WAR" OR "LANDMINE" OR "EXPLOSIVES" OR "UNEXPLODED ORDNANCES" AND "AMPUTATION" OR "ARTIFICIAL LIMB" OR "INJURY" AND "REHABILITATION" OR "THERAPY" AND "COMMUNITY" OR " SUPPORT NETWORK") OR ("VICTIMS" OR "SURVIVORS" AND "LANDMINES" OR "ANTIPERSONNEL MINE" AND "REHABILITATION" OR "CBR" OR "REHABILITATION IN COMMUNITY") OR ("WAR" AND "AMPUTATION" OR "INJURY" AND "LANDMINES" OR "ANTIPERSONNEL MINE" OR "UNEXPLODED ORDNANCES" AND "REHABILITATION" OR "EFFECTS") OR ("WAR" AND "AMPUTATION" AND "REHABILITATION" AND "SOCIAL SUPPORT" OR "COMMUNITY")

Figura 2. Ecuación general de búsqueda 


\section{Referencias}

Dodge, B. (2009). Motores de búsqueda y Álgebra Booleana. Estados Unidos: Biblioteca de la Universidad de Albany.

ELSEVIER. (2017). ELSEVIER. Obtenido de ELSEVIER: http://www.americalatina.elsevier.com/corporate/es/scopus.php

Isaacson, B.; Hendershot, B. ; Messinger, S.; Wilken, J. ; Ràbago, C. ; Esposito, E.; Wolf, E. ; Pruziner, A. (2017). The center for rehabilitation sciences research: Advancing the rehabilitative care for service members with complex trauma.

Nagles, G. (2007). LA GESTIÓN DEL CONOCIMIENTO COMO FUENTE DE INNOVACIÓN. Revista Escuela de Administración de Negocios, 78.

Ordóñez, G.; Hernández, A.; Hernández, C. \& Méndez, C. (2009). Análisis bibliométrico de la Revista de Economía Institucional. Revista de Economía Institucional. p 309-353.

Vantage Point. (2018). Vantage Point. Obtenido de Vantage Point: https://www.thevantagepoint.com/

Veale, D.; Gilbert, P.; Wheatley, J.; Naismith, I. (2014). A New Therapeutic Community: Development of a Compassion-Focussed and Contextual Behavioural Environment. Rewiev Social Psychology, $p 45$.

Vargas, F. C. (2005). Vigilancia como elemento de innovación y desarrollo tecnológico. Ingeniería en investigación, p 58.

Walsh, N. ; Walsh, W. (2003). Blast injury; Cost of illness; Economic aspect; Health care cost; Landmine; Length of stay; Limb prosthesis; Major clinical study; Social aspect; Victim; weapon. 